\title{
Study on the Design and Implementation of the Mobile Terminal \\ Real-Time Communication System
}

\author{
Yanping Wei ${ }^{1}$, Hailiu Xiao ${ }^{1}$ \\ ${ }^{1}$ Nanchang Institute of Science and Technology, Nanchang, Jiangxi, 330108 \\ 346591653@163.com
}

KEYWORDS: Mobile Terminal System; Real-time Communication; Implementation

\begin{abstract}
Instant messaging technology in the mobile Internet has been widely recognized, the relationship more closely to user requirements can be more real-time multimedia communications, which requires a network connection in the mobile Internet is not very stable environment and provide a reliable instant communication mechanism. At present, both at home and abroad has been very mature instant messaging software, but developers are conservative with their own communication protocol and related communications technologies. Therefore, this article from the actual needs of broadcast interactive phone system, aiming at low-power mobile devices, the small memory capacity and other characteristics, Shenyang broadcast television users, based on the use of mobile phone users to broadcast existing social relationships, combining broadcast functional Wechat and micro-blogging and other interactive, design and implement a mobile social network based instant message notification communication mechanism.
\end{abstract}

\section{Introduction}

As ICT and Internet development and progress, promote the establishment of social networks, the knowledge and application of the rapidly changing information age, people of their requirements continue to increase, which drives the development of social networks, and social networks continue meet people for social interpersonal needs. It can be a variety of fixed terminals and mobile terminals, the use of social networking applications, anytime, anywhere, whenever they want to contact with anyone, the reality of social interaction for people to save a lot of space and time, but also to promote better social networks development of. The rapid development of mobile Internet and the popularity of social networking is a necessary foundation for development, but also to the development of social networks presents a new trend, the specific performance of mobile and social networking niche. Mobile social networking technology is the customer end more run on mobile devices, anytime, anywhere access to more mobile Internet applications and provides location information provided by GPS and LBS socialize; a small minority representation in the social network is no longer It is for all users of social popularity, but for specific populations and the establishment of social platforms, such as enterprise social networking platform, shopping and other social networking platforms.

\section{Mobile Social Network Instant Communication Mechanism}

Most IM systems are using the system architecture of a client / server architecture. For server provider and protocol designers fundamental question is how to assign users to connect to each 
server fair, the number of users and the use of IM software changes over time, how can we ensure that the number of users peaked the organizational structure of the system is still able to adapt to the changes and adjustments. Currently, there are two general system architectures: symmetric and asymmetric architecture. Symmetric architecture refers to all functions required to service all of the servers, rather than symmetrical structure by different servers different roles, together to complete all the features. For symmetric architecture instant messaging system, client does not need to distinguish between the servers, only need to connect to either a server can complete all requirements. For the purposes of asymmetric instant messaging system, different servers assume different roles, depending on the roles were completed, such as message forwarding, a user logs on, the language services and other functions. This project uses a symmetric structure system architecture, users can connect to any one of the broker. In the instant messaging system, there is an important issue is communication data transmission format. Currently, forms of data transmission formats are basically two. Many protocols transmit data in binary format using the network byte order, including TCP and IP protocols, and for HTTP, SMTP and POP3 and other application layer protocols use format for transmission of information in text form. Binary data transmission can often speed up data processing speed and can save network resources, but for people who understand text-based form of agreement and easy to view, analyze, and debug. The project is based MQTT protocol, which uses the text in the form of a packet format. For the transmission of a text message, in addition to communication architecture is based on C / S mode, the message transmission are built on the basis of the TCP protocol, and the server will generally provide a fixed port to provide services, the server provides a fixed service port passive communication with the client, play the role of message transfer.

\section{Design of Instant Communication Interface}

IM Some of the information is obtained from the social network, such as instant messaging between users send may contain tweets, which requires users can access social networks provide interface information. Built in ELGG in the WebService framework, but it is only the frame, we need to define their own needs according to the relevant service interfaces. Use WebService process, ELGG provides two authentication mechanisms: simple KEY and HMAC two kinds. Here KEY simple manner. By way of plug-ins can also be used similar Weibo OAuth authentication method, will not consider here. KEY Keyadmin generated through the use of plug-ins to complete the public and private key generation. Client through public key authentication API for user authentication auth_token, complete the user login process. Defined here WebService interface is divided into several types of interfaces: user interfaces class information, micro-blogging interface that topic class interface, private letters class interface, user information, modify the interface that the user's friends class interface, the user interface to modify the password, the user class recommended interface.

User information class interface. 1. Obtain user authentication information 1) Interface Name: auth. gettoken; 2) the required parameters: username: user name, required parameters; Password: user password required parameters; 3 ) type of call: POST; 4) returns the result: Array ([status] => 0 [result] => 5b890e8c44cf084077430092e1e82f20); Note: array, result is the corresponding user authentication information. 2. User registration; 1) Interface Name: user. register; 2) the required parameters: name: user name, required parameters; email: e-mail address, required parameters; username: user login name, required parameters; password: user's password, required parameters; api_key: client key, required parameters; 3) type of call: POST4) returns the result: Array ([status] $=>0[$ result $]=>$ Array $([$ success $]=>1$ [guid] => 444) ) or Array ([status] $=>0$ [result] => Array 
([success] $=>$ [message] => That username already exists)) or Array ([status] $=>0$ [result] => Array ([success ] $=>$ [message] $=>$ That name already exists)) or Array ([status] $=>-1$ [message] $=>$ Method call failed the API Authentication) NOTE: On success, returns for a new guid registered user ID information. If the registration fails, the information will be given in the message. If authentication fails, it will give the corresponding description.

\section{Design and Implementation of Real-Time Communication Mechanism}

The server system used Linux as a development platform, Linux is a free operating system, the system is simple, stable, high efficiency, support for select, poll, epoll and other efficient event-driven network server model. Because MQTT written in C and based on experience in the development project team members, most of the system using $C$ language development, $C$ language compiler efficiency with high efficiency, and is suitable for the preparation of efficient network server. Choose Nginx instead of Apache as the Web server to MySQL database-based, Reids database supplement. Some topics are server automatically for the user to subscribe, users can also take the initiative to subscribe to topics of interest to the broker when the server receives a message on a topic, users can subscribe to these topics push messages. Topics are divided into two categories: system and user class topics like the topic. System class topics, primarily for push notification system, it is recommended to provide technical information and advertising support.

Presence is in the form of topic to achieve, it is divided into three topics: the user's state information presence status, the user's personalized signature presence / signature and the name of the user is listening to the frequency of presence / listening. There are online user status information, offline, busy and away from other states. By the client automatically if the subscription line on each user presence topic, the user complex relationship, it will affect the performance of the client. In order to improve client performance, reduce network resource occupied by the mobile terminal, the server will automatically subscribe users to the presence of mutual concern buddy topic, therefore, when the user logs on to the server does not need to turn all your friends subscribe to the friends list of presence information. Information such as the user's buddy list is stored locally, when a user logs on, the server will obtain presence information for the current user's buddy list by viewing the database will be the current user's friends' status, personal signature, and the name of the user is listening frequency sequentially reply to the user. Some users buddy presence information changes, it will send a presence update message to the server, the server by matching the current online users and their friends, send presence updates.

After the user logs in the system, between the client and server to establish a long MQTT connection (MQTT is already a long socket connection), to establish a connection between the media server and you need to send multimedia messages. In the speech, for example, when sending voice information between users friends, this time to establish the connection between the user and the voice server will send a voice message to a multimedia server, the server returns the media link information, user information is encapsulated in a message sent this link to MQTT server, MQTT server parses the message header, this link information is sent to the user to be sent to the user. User and the user's friends want to get voice messages are by clicking on the link to get the multimedia server. The server returns a link to the format pathname / file name, the only link requirements, mainly reflected in the file name, unified directory under the same name as the file is not allowed. File path into year / month / day three. For an image, according to the requirements of the original image storage medium and small format, the original image size is larger view, $75 \%$ of the original size of the map, the name is based on the big picture plus medium.jpg, $50 \%$ of the original size a small map, the name is based on the big picture plus small.jpg. This allows the client to display the 
appropriate size image, when users click on image to download the original image on the server. For small-time recording and picture transmission can not tell the length of the file server, the server according to the end to determine whether the flag has been received. For the type of media file, required to support disconnected resume, you need to tell the server file size information transmission, the breakpoint is based on the time of the transfer file size to decide whether the receiving end is judged based on file size. Breakpoint information is stored in a MySQL database, each user uploading a video for the first time, it will insert a corresponding message in the database records the user name, file name, file upload and location. Users to upload, it will first check the availability of the database file relevant records, if any, from the breakpoint continue to upload, upload successful saved on the server, then delete this error message. The server responds to the client's need for timely progress of the upload.

\section{Conclusion}

Most important feature of instant messaging software on the Internet is real-time exchange of information. It produces a profound social and technical reason. Almost all people have the desire to socialize, access to social respect, self-realization of the demand, which is the driving force of popular Internet instant messaging software. And increasingly developed material civilization of the side effects, but also makes people used to keep a distance from the people around, so people are more willing to open their hearts to strangers. Compared with the traditional means of communication, instant messaging is fast, cheap, hidden features in the network across age, identity, industry, geographical constraints, to achieve zero distance interpersonal communication, between people and information. In this sense, there is a network instant messaging has changed the way people communicate and dating culture, greatly expanding the space for the exchange of personal life.

\section{REFERENCE:}

[1] Y. Lu, T. Zhou, B. Wang. Exploring Chinese users' acceptance of instant messaging using the theory of planned behavior, the technology acceptance model, and the flow theory [J]. Computers in Human Behavior, 2009, 25 (1): 29-39.

[2] J.-S. Leu, Y.-P. Chi, W.-K. Shih. Design and implementation of Blog Rendering and Accessing Instantly system (BRAINS) [J]. Journal of network and computer applications, 2007, 30 (1): 296-307.

[3] K. K. Sharma, R. B. Patel, H. Singh. A Reliable and Energy Efficient Transport Protocol for Wireless Sensor Networks [J]. International Journal of Computer Networks \& Communications, 2010,2 (5):

[4] N. Garrett, B. Thoms, M. Soffer, T. Ryan. Extending the ELGG social networking system to enhance the campus conversation [J]. Second Annual Design Research in Information Systems (DESRIST), Pasadena, California, 2007,14-15. 